Edición Extra-Ordinaria. ISSN 2027-1034 P. p 446 - 445

Memorias del VII Encuentro Nacional de Experiencias en la Enseñanza de La Biología y la Educación Ambiental y II Congreso Nacional de Investigación en la Enseñanza de la Biología

\title{
LAS PRÁCTICAS DE CAMPO UNA ESTRATEGIA DIDÁCTICA PARA CONSERVAR EL ECOSISTEMA DE PÁRAMO DESDE ELESTUDIO ECOFISIOLÓGICO DEL FRAILEJÓN (ASTERACEAE: ESPELETIA).
}

\section{FIELD PRACTICE AS A TEACHING STRATEGY TO PRESERVE THE MOOR ECOSYSTEM FROM THESTUDY OF FRAILEJÓN ECOPHYSIOLOGICAL (ASTERACEAE: ESPELETIA).}

\section{Maryury Fernanda Gómez Barrera ${ }^{1}$}

\section{Resumen}

Esta investigación destaca la importancia del maestro de ciencias como promotor de actitudes, conocimientos y prácticas hacia la conservación del ecosistema páramo a través de las prácticas de campo, en estudiantes de secundaria del Colegio Siervas de San José, como un acercamiento a la conservación de los ecosistemas Colombianos.

Recurriendo para ello al estudio ecofisiológico de una planta concreta, endémica y de gran significado ecológico y social para el ecosistema páramo, el frailejón (Asteraceae: Espeletia). La investigación presenta un enfoque mixto o multimodal, recurriendo a la IEP (Investigación como Estrategia Pedagógica) como metodología central y fundamental para el desarrollo de esta investigación, se logran describir las nociones de las estudiantes en cuanto a la conservación, la ecofisiología, el páramo y el frailejón; y se caracterizaron algunas actitudes, prácticas y conocimientos conservacionistas de las estudiantes en cuanto al páramo evidenciando las transformaciones durante el proceso.

Palabras claves: páramo, frailejón, prácticas de campo, conservación, ecofisiología.

\section{Abstract}

This research highlights the importance of science teacher in promoting attitudes, knowledge and practices to moor ecosystem conservation through field practice in high school students Colegio Siervas de San José, as an approach to conservation Colombian ecosystems.

By studying a particular plant ecophysiological, endemic and high ecological and social significance to the ecosystem moor, the frailejón (Asteraceae: Espeletia). The research presents a mixed approach or multimodal, using the IEP (Research as a Pedagogical Strategy) as central and fundamental methodology for the development of this research, we are able to describe the concepts of students regarding conservation, ecophysiology, the moor and frailejón, and characterized some attitudes, practices and knowledge students

${ }^{1}$ Estudiante tesista de Licenciatura en Biología, Universidad Pedagógica Nacional. 2013. marif 16g@hotmail.com o dbi823 mgomez@pedagogica.edu.co 
Bio-grafía Escritos sobre la Biología y su Enseñanza.

Edición Extra-Ordinaria. ISSN 2027-1034 P. p 446 - 445

Memorias del VII Encuentro Nacional de Experiencias en La Enseñanza de La Biología y la Educación Ambiental y II Congreso Nacional de Investigación en la Enseñanza de la Biología

conservationists as the moor showing the transformations during the process.

Keywords: moor, frailejón, practical field, conservation, ecophysiology.

\section{Introducción}

El maestro que enseña biología tiene la cualidad de provocar grandes intereses, aficiones y rechazos en los estudiantes hacia el estudio de lo vivo, reconocer a este profesional de la educación como promotor de actitudes hacia la comprensión de lo vivo y las interacciones que todos los organismos presentan entre sí y su ambiente, permitirá que los estudiantes identifiquen y conozcan parte de la biodiversidad de su país y progresivo a ello establezcan las amenazas que enfrenta esta. Por lo tanto, aproximarse a reconocer la diversidad biológica de Colombia es el primer paso para ejercer acciones de protección y conservación del conjunto de especies y comunidades biológicas que se encuentran en gran devastación (Primack, 2010).

Reconociendo la escuela como una generadora de cambios en el pensar y el actuar de las personas y al maestro como quien permite avanzar en el conocimiento de la ciencia y en la enseñanza de esta, siendo un sujeto investigador que recurre a la investigación como un recurso para innovar y transformar los procesos educativos, incentivando la renovación de las prácticas cotidianas las cuales se configuran en gran parte en la escuela y el maestro resulta crucial en el desarrollo de éstas.

Es por esta razón que un sujeto que enseña y participa en el estudio de lo vivo desde el ámbito investigativo puede aportar significativamente en la formación de sujetos críticos e investigadores, donde la experiencia que brindan este tipo de actividades de enseñanza de las ciencias se constituye entre el maestro y los estudiantes al conocer, interactuar y poner a prueba investigaciones que parten desde el interés de estos actores con el fin de reconocer la biodiversidad y a su vez conservarla.

Para este fin, se parte desde el aprendizaje por experiencia (Dewey, 2004) el cual desarrolla procesos de pensamiento, que van de la mano de la observación, investigación, indagación y exploración, recurriendo a las prácticas de campo como una excelente estrategia didáctica para aprender y enseñar a conservar desde la experiencia misma; puesto que las prácticas de campo como lo menciona Amórtegui y Correa (2012) son una herramienta indispensable que generan nuevos conocimientos 0 actúan como complemento y refrendación de los elementos teóricos dados en clase.

En este sentido, se busca promover la conservación de un ecosistema clave y de fácil acceso para los estudiantes bogotanos, el Páramo que cuenta con especies endémicas y cumple una función vital para la regulación del ciclo hidrológico del país, que a su vez es víctima de gran variedad de disturbios antrópicos y de allí su necesidad de educar en pos de la conservación de este. 
Edición Extra-Ordinaria. ISSN 2027-1034 P. p 446 - 445

Memorias del VII Encuentro Nacional de Experiencias en La Enseñanza de La Biología y la Educación Ambiental y II Congreso Nacional de Investigación en la Enseñanza de La Biología

Además, el páramo albergar una gran diversidad de fauna y flora, entre ella los frailejones especies simbólicas de este ecosistema que son abundantes y por sus características morfológicas permiten comprender las características ecológicas de este.

Destacando a las prácticas de campo como una excelente estrategia para desarrollar habilidades en el uso de procedimientos científicos, motivar al estudiante, ayudar a la comprensión de los planteamientos teóricos y promover actitudes relacionadas con el conocimiento científico (Carmen, 2000 citado por Amortegui y Correa, 2009) en general y la biología en particular.

Surge entonces, la necesidad de plantear salidas de campo que indaguen sobre el ecosistema de Páramo y que profundicen sobre el frailejón (Espeletia), de manera que se estudie y fomenten actitudes en los estudiantes hacia la conservación de este ecosistema y su comprensión desde el estudio ecofisiológico de esta planta, el cual permite estudiar el organismo y su ambiente; comprendiendo la diversidad fisiológica en función del ambiente en el que habitan los organismos para saber el estado de su hábitat identificando los esfuerzos conservacionistas que deben ejecutarse, promoviendo el trabajo en campo y la investigación científica como un modo de conocer lo vivo desde la experiencia de investigar, observar y proponer acciones a problemáticas de los ecosistemas.

A partir de esto, se plantea como tema problema y objetivo de esta investigación, desarrollar conocimientos actitudinales, procedimentales y conceptuales para la conservación del ecosistema Páramo a través de las prácticas de campo, en estudiantes del colegio Siervas de San José a partir del estudio ecofisiológico del frailejón (Asteraceae : Espeletia).

\section{Metodología}

El paradigma que trabaja esta investigación es el crítico social, el cual pretende superar el reduccionismo y el conservadurismo admitiendo la posibilidad de una ciencia que no sea ni puramente empírica ni sólo interpretativa, y sobre todo que ofrezca aportes para el cambio social desde el interior de las propias comunidades. Su finalidad es transformar la estructura de las relaciones sociales y dar respuesta a determinados problemas generados por éstas, partiendo de la acción-reflexión de los integrantes de las comunidades y de su participación (Alvarado y García, 2008).

El enfoque investigativo que se presenta para el desarrollo de este Trabajo de Gradoesmixto o multimodal, el cual es la fusión de los enfoques de investigación cualitativo y cuantitativo, ya que ambos enfoques, utilizados en conjunto, enriquecen la investigación, y permiten mejor comprensión del fenómeno en estudio y a su vez, mejor precisión otorgándole validez y confiabilidad a los datos; Mezclar estos dos modelos permite tal como lo menciona Hernándezet al (2003), potenciar el desarrollo del conocimiento, la construcción de teorías y la resolución de problemas; ya que la investigación 
Edición Extra-Ordinaria. ISSN 2027-1034 P. p 446 - 445

Memorias del VII Encuentro Nacional de Experiencias en La Enseñanza de La Biología y la Educación Ambiental y II Congreso Nacional de Investigación en la Enseñanza de La Biología

cuantitativa ofrece la posibilidad de generalizar los resultados ampliamente, brindando la posibilidad de comparar estudios similares; por su parte, la investigación cualitativa da profundidad a los datos, riqueza interpretativa, aportando un punto de vista holístico, buscando entender el contexto y el punto de vista del actor social.

Como método de investigación, se utiliza la Investigación como Estrategia Pedagógica (IEP) la cual surge dentro del Programa Ondas (Colciencias, 2012), este método permite la divulgación de las prácticas que generan cambios de sentidos en las realidades de los individuos y promueve nuevas valoraciones críticas de su cotidianidad, donde la investigación toma un papel trascendental para la formación de niños (as), jóvenes, adultos, maestros (as). Posibilitando a los estudiantes apropiarse de las lógicas del conocimiento y de herramientas propias de la ciencia, pero sobretodo, de una mirada de la sociedad y sus asuntos. Esta estrategia pedagógica reconoce en los niños y jóvenes su capacidad para explorar, observar y preguntar, desarrollando habilidades y capacidades, compartiendo saberes y conocimientos, todo ello mediante la experiencia y la vivencia de hacer investigación, lo que implica aprender haciendo; favoreciendo el aprendizaje por descubrimiento y el aprendizaje significativo en cada uno de los estudiantes, debido a que al investigar el aprendiz se reconoce y descubre otras realidades, se relacionan con el entorno y busca modificarlo, establece relaciones, distingue causas y consecuencias y adquiere conocimientos con sentido.

\section{Fases de la investigación}

Para llevar a cabo el desarrollo de esta investigación se organizaron 4 fases, las cuales fueron encaminadas a cumplir cada uno de los objetivos planteados, desarrollando la consolidación del grupo de estudiantes a trabajar, la descripción de sus preconceptos, las sesiones de clases, las salidas de campo y la transformación actitudinal y formación de conceptos y procedimientos, en cuanto al páramo, su conservación y el funcionamiento de este y de los organismos que en este ecosistema habitan a partir del Frailejón.

\section{FASE I Contextualización}

En esta etapa se buscó reconocer la población y el lugar en el cual se ejecutó el proyecto, para esto se realizo la revisión de documentos de la institución educativa, tales como el recuento histórico y enfoque del colegio (Misión. Visión), el PEI (Plan de Estudios), PRAE (Proyecto Ambiental Escolar) y el currículo del Área De Ciencias Naturales.

A su vez, el grupo de estudiantes con quienes se realizó todo el proyecto investigativo fue el Grupo Ambiental de la institución, siendo este trabajo investigativo como un apoyo al PRAE. Dicho grupo fue contextualizado junto con el Colegio, por medio de la observación y la inmersión dentro de las 
Edición Extra-Ordinaria. ISSN 2027-1034 P. p 446 - 445

Memorias del VII Encuentro Nacional de Experiencias en la Enseñanza de La Biología y la Educación Ambiental y II Congreso Nacional de Investigación en la Enseñanza de La Biología

dinámicas educativas de la institución, permitiendo acercamiento con el grupo de investigación.

\section{FASE II Diagnostico de conceptos previos y actitudes hacia la conservación}

Esta etapa se caracteriza por la aplicación de un cuestionario que permite identificar las nociones de las estudiantes en cuanto a la conservación, la ecofisiología, el páramo y el frailejón, para esto se diseñaron preguntas abiertas como: la conservación se refiere a, un frailejón es, la ecofisiología se ocupa de, un páramo es.

A su vez en esta segunda fase se desarrolla un test organizado en escala tipo Likert sobre las razones para conservar el páramo, teniendo una visión preliminar de las actitudes del grupo de trabajo hacia la conservación; dicho test se implementa al finalizar todo el proceso con el fin de evidenciar si ocurrieron o no transformaciones en las actitudes de las estudiantes hacia el páramo.

\section{FASE III Aproximación al ecosistema paramuno}

Luego de reconocer los conocimientos previos que tienen los estudiantes y sus actitudes frente al páramo y su conservación, se dio paso a esta fase, la cual se desarrolla a partir de:

- Sesiones de clase, apoyadas por lecturas, resúmenes de las clases, material virtual y trabajo de consulta.Donde se trabajaron en once (11) sesiones temas como: principales ecosistemas de Colombia, ecosistemas cercanos o dentro de Bogotá, páramo y su flora (frailejón) y fauna; estructuras adaptativas en las plantas de páramo, la ecofisiología en el páramo, estrés en las plantas, conservación. Temas y conceptos necesarios para la ejecución de las salidas de campo.

- Prácticas de campo,donde todo el trabajo de aula se acciona, permitiendo acercamiento y desarrollo de actitudes a fines al páramo; se desarrollaron dos salidas de campo, la primera al Jardín Botánico José Celestino Mutis con el fin de conocer la flora de los principales ecosistemas de Colombia, identificando sus características morfológicas y condiciones ambientales, con el fin de compararlas con el páramo. Y al Páramo de Chingaza, donde se identificaron las características del ecosistema (suelo, clima, vegetación...) y el estudio ecofisiológicos de los frailejones (Espeletia grandiflora, EspeletiauribeiyEspeletiaargentea).

\section{FASE IV Caracterización y transformación de las actitudes, prácticas y conocimientos}

Después de comprender las dinámicas del ecosistema, conocer su flora y fauna y desarrollar la caracterización ecofisiológicas de las especies de frailejón presentes en el páramo de Chingaza. Se realizaron trabajos 
Edición Extra-Ordinaria. ISSN 2027-1034 P. p 446 - 445

Memorias del VII Encuentro Nacional de Experiencias en La Enseñanza de La Biología y la Educación Ambiental y II Congreso Nacional de Investigación en la Enseñanza de La Biología

individuales (como ensayos) y grupales (como $V$ heurística) con el fin de evidenciar los conocimientos, actitudes y practicas formadas en este proceso, junto con la aplicación por segunda vez de Test razones para conservar el páramo, comparándolo con el primer test y evidenciando sí se desarrollaron conocimientos actitudinales, procedimentales y conceptuales para la conservación de dicho ecosistema con el ánimo de validar todo el proyecto investigativo.

\section{Resultados y discusión}

El Colegio Siervas de San José, es un colegio religioso fundado por la congregación Siervas de San José dedicado a la educación de mujeres, procurando generar estudiantes con un pensamiento analítico-crítico-creativo. Se encuentra ubicado en la zona urbana del barrio la Calleja, en la localidad de Usaquén, situado al norte del Distrito Capital. La institución busca ser reconocida por formar ciudadanas que cumplan con los objetivos cristianos y humanos, de compartir, servir, colaborar, transformar, liberar, siendo personas que defiendan y amen la vida (PEI, 2002).

El proyecto investigativo se desarrolló con las estudiantes que pertenecen al "grupo ambiental" de la institución, siendo un grupo de alta heterogeneidad que está conformado por quince (15) estudiantes de los grados sexto, séptimo, noveno y décimo, el cual hace parte del desarrollo del PRAE, que tiene como findespertar y desarrollar en todos los miembros de la comunidad educativacompromiso en torno a la conservación, protección y mejoramiento del Ambiente, formando personas autónomas, responsables y propositivas, haciendo uso racional de los recursos naturales, y mejorar nuestra calidad de vida (Benavides et al, 2013).

A partir del cuestionario de conceptos previos y la primera aplicación del Test, se identificó que el $40 \%$ de las estudiantes saben que es un ecosistema, el $40 \%$ aproximadamente compre la conservación como una forma de protección de las especies y sus ecosistemas; $66 \%$ de las estudiantes reconocen al frailejón como una planta que se encuentra en los páramos, $6 \%$ se aproxima al objeto de estudio de la ecofisiología y el 100\% del grupo reconoce al páramo como un lugar frio, húmedo y de reserva de agua. En cuanto al test de razones para conservar, se plantearon 10 situaciones en las que se buscada identificar actitudes de las estudiantes en cuanto a la importancia y conservación del páramo (Educación, ciencia, consumo, turismo, medicina, base ambiental, servicios ecosistémicos, valor intrínseco y cultura humana) teniendo tres alternativas para responder (totalmente en desacuerdo, parcialmente de acuerdo, totalmente de acuerdo).

En la primera sesión de clase se aplica este Test, en el cual se identifica que $86 \%$ de las estudiantes reconocen que el páramo debe protegerse por los servicios ecosistémicos que este ofrece, el $73 \%$ reconoce el valor intrínseco de 
Edición Extra-Ordinaria. ISSN 2027-1034 P. p 446 - 445

Memorias del VII Encuentro Nacional de Experiencias en La Enseñanza de La Biología y la Educación Ambiental y II Congreso Nacional de Investigación en la Enseñanza de La Biología

este ecosistema y la necesidad de estudiar los organismos que allí viven. Mientras $53 \%$ se muestran en desacuerdo a la conservación del páramo por razones de consumo y el $46 \%$ encuentra al turismo como una razón para la conservación. Estos datos permiten identificar parcialmente al grupo y sus razones para conservar o no la biodiversidad de ecosistemas, tales como el páramo.

Consecutivo a ello, en las sesiones de claseserealizó un trabajo masivo con las estudiantes evidenciando a partir de las consultas, preguntas en clase, ensayos y demás, la capacidad argumentativa fomentada, donde el uso de los conceptos y la comprensión de estos se hicieron explícitos de forma verbal y escrita; aditivo a ello las dos prácticas de campo, su planeación y realización que actúan de modo indispensable para comprender las dinámicas de los organismos y sus ambientes permitieron en conjunto reconocer que sí es viable la implementación de trabajos prácticos en las enseñanza de la biología como una estrategia didáctica hacia la conservación de ecosistemas. Evidenciándose en la aplicación del Test por segunda vez, que las estudiantes en un $60 \%$ ven la importancia de la conservación por razones científicas, se hace más sólida la actitud de no promover el consumo de los páramos (86\%), mientras que el $73 \%$ afirma que debe primar las culturas humanas y sus relaciones con los ecosistemas antes que las actividades mineras; en general se evidencia un fortalecimiento de las actitudes afines hacia la conservación, así como el desarrollo de conocimientos actitudinales, procedimentales y conceptuales para la conservación del ecosistema páramo, que se cuantifican en el test, pero se evidencian de modo cualitativo en todas las actividades ejecutadas en las clases, prácticas de campo, informes y actividades de divulgación a la comunidad educativa.

Reflejándose en esta investigación la importancia de promover la investigación biológica y educativa en las instituciones y la necesidad de acercar a los estudiantes a los ecosistemas, pues no siempre las fotografías o el uso de materiales virtuales resultan amenos y llamativos al estudiantado, pues como lo menciona John Dewey (2004) "Una onza de experiencia esmejor que una tonelada de teoría."

\section{Conclusiones}

El hombre y la montaña es una relación que constituye un capítulo en la historia de la humanidad casi tan viejo como el hombre mismo. Precisamente en Colombia no hay que hacer mucho énfasis para explicar esta situación, aproximadamente el $70 \%$ de la población vive y trabaja en la montaña. Es más, lo que fue de este país, lo que es en el presente y lo que va a ser en el futuro, depende de la actitud que el hombre asuma frente a las montañas, porque lo demás es complemento. (Guhl, 1982)

Siendo la escuela, el maestro y los estudiantes actores fundamentales para comprender lo vivo, sus peligros y plantear desde la experiencia misma métodos para conservar y mitigar el daño a los ecosistemas. Esta investigación 
Edición Extra-Ordinaria. ISSN 2027-1034 P. p 446 - 445

Memorias del VII Encuentro Nacional de Experiencias en La Enseñanza de La Biología y la Educación Ambiental y II Congreso Nacional de Investigación en la Enseñanza de La Biología

muestra la viabilidad de fortalecer y desarrollar actitudes, conocimientos y procedimientos hacia la conservación, ya que estos tres en conjunto resultan ser trascendentales a lo largo del proceso educativo pues permite analizar los comportamientos que se "deberían" saber, actitudes que se "deberían" pensar y prácticas que se "deberían" realizar, entendiendo porque la gente hace lo que hace.

Comprendiendo la importancia de las Prácticas de campo en la formación del estudiantado, siendo una estrategia que permite mejorar el proceso de enseñanza/aprendizaje no solo de la biología, sino que a su vez permite la formación de ciudadanos que reconozcan la diversidad biológica y cultural de su país y la necesidad de conocerlo para así conservarlo.

\section{Bibliografía}

- $\quad$ ALVARADO, L. y GARCÍA, M. (2008).Características más relevantes del paradigma socio-critico: su aplicación en investigaciones de educación ambiental y de enseñanza de las ciencias realizadas en el Doctorado de Educación del Instituto Pedagógico de Caracas. Sapiens, Revista Universitaria de Investigación, año 9, No 2, Diciembre.

- AMORTEGUI,E. y CORREA,M. (2012).Las prácticas de campo planificadas en el proyecto curricular de la Licenciatura en biología. Universidad Pedagógica Nacional. Caracterización desde la perspectiva del conocimiento profesional del profesor de Biología. Universidad Pedagógica Nacional, fundación Francisca Radke. Bogotá.

- AMORTEGUI, E. y CORREA, M. (2009).Las prácticas de campo planificadas en el proyecto curricular de la Licenciatura en biología. Universidad Pedagógica Nacional. Caracterización desde la perspectiva del conocimiento profesional del profesor de Biología. Tesis de Grado Universidad Pedagógica Nacional.

- BENAVIDES, BOHÓRQUEZ, DIAZ, GÓMEZ Y GUTIERREZ. (2013). Proyecto ambiental escolar Colegio Siervas de San José. Documento inédito, Bogotá, Colegio Siervas de San José.

- COLCIENCIAS.(2012).La Investigación como estrategia pedagógica. Consultado el día 8 de Agosto del 2013, de la página web: http://www.colciencias.gov.co/wiki ondas/la-investigaci-n-comoestrategia-pedag-gica-iep

- DEWEY. (2004).Experiencia y educación. Madrid: Biblioteca nueva.

- GUHL. E. (1982). Los páramos circundantes de la sabana de Bogotá. Jardín Botánico José Celestino Mutis. Bogotá: litografía arco. 
Bio-grafia Escritos sobre la Biología y su Enseñanza.

Edición Extra-Ordinaria. ISSN 2027-1034 P. p 446 - 445

Memorias del VII Encuentro Nacional de Experiencias en la Enseñanza de

La Biología y la Educación Ambiental y II Congreso Nacional de Investigación en la Enseñanza de la Biología

- HERNÁNDEZ R., COLLADO C. y BAPTISTA P. (2003).Metodología de la investigación. México: Mc Graw Hill.

- PEI. (2002). Proyecto Educativo Educacional Colegio Siervas de San José. Unilibros, Bogotá D.C.

- PRIMACK.R.B. (2010). Essentials of Conservation Biology. (Fifth Edition).Sinauer Associates, Inc., Sinderland, Massachusetts. Traducido y Adaptado por Rodrigo Torres Núñez. Biólogo MS.c Profesor Asociado, Departamento de Biología, Universidad Pedagógica Nacional. 